\title{
ÉTUDE D'UNE RÉACTION PÉROXYDASIQUE APPROPRIÉE \\ POUR LA DÉTECTION DE L'EAU OXYGÉNÉE DANS LES LAITS DE CONSOMMATION
}

\author{
par \\ A. EYRARD \\ R. J, JOUFFRET \\ Directeur \\ Directeur \\ du Laboratoire des Groupements Interprofessionnels Laitiers \\ Paris \\ Rouen
}

\section{Introduction}

Une politique de qualité du lait de consommation doit proserire l'utilisation d'antiseptique, d'eau oxygénée en particulier, contraire aux règlements d'hygiène publique et autorisant une politique du moindre effort. L'exemple similaire de l'industrie beurrière où la tolérance de l'acide borique eomme conservateur a été trop longtemps admise, montre combien de telles pratiques sont néfastes pour la qualité du produit, en paralysant tout progrès techniquee. Par ailleurs, la valeur hygiénique des laits de consommation étant contrôlée par l'analyse bactériologique, il importe de vérifier préalablement à tout contrôle, que le lait est exempt d'eau oxygénée.

On sait que l'eau oxygénée ajoutée au lait, toujours à très faibles doses, 2 à $4 \%$ o, est décomposée plus ou moins rapidement suivant le mode de conservation et que les méthodes chimiques classiques sont impuissantes à en permettre la détection. Il faut donc avoir recours à des méthodes plus sensibles en s'adressant en particulier à des réactions biochimiques. Cela avait conduit, en 1928, M. TAPERNoUx [1] à rechercher l'eau oxygénée par la réaction classique de Dupouy. Par cette méthode, M. TAPERnoux caractérisait l'eau oxygénée ajoutée au lait, pendant les trois premières heures postérieures à son addition dans le cas du lait cru, et pendant dix à vingt heures après son adjonction au lait pasteurisé. Ces résultats, déjà fort intéressants, sont néanmoins insuffisants dans le cas des laits pasteurisés livrés à la consommation au plus tôt vingt-quatre heures après la pasteurisation, comme c'est le cas pour les laits pasteurisés au lieu de production et vendus à Paris.

Nous nous sommes proposés, dans ce travail, d'établir un contrôle pratique permettant de caractériser ávec certitude l'addition d'eau oxygénée dans le lait destiné à la consommation d'un grand centre.

\section{Etude comparative des réactions peroxydasiques}

Nous avons été amenés à étudier comparativement les différentes réactions peroxydasiques caractéristiques tant en ce qui 
concerne la fidélité de leurs indications que leur sensibilité relative vis-à-vis de la diastase active, d'une part, et du peroxyde, d'autre part.

Nous avons étudié les réactions de Dupouy [2], Storch [3], Rotenfusser [4] et Schern-Schellhaze [5].

La sensibilité par rapport à la diastase active a été étudiée en ajoutant, à du lait préalablement débarrassé de peroxydase active par chauffage, du lait cru en proportions déeroissantes jusqu'à une limite inférieure prise comme test. La sensibilité ainsi définie est donc exprimée en pourcentage de lait cru décelé par la réaction étudiée. Tous les essais rapportés ci-après ont été effectués avec de l'eau oxygénée à 10 volumes.

Les résultats de ees essais sont rassemblés dans le tableau I ci-après :

\section{TABLEAU I}

SENSIBILITEESS ET FIDELLITÉS COMPAREES DES DIFFÉRENTES REACTIONS

\begin{tabular}{|c|c|c|c|}
\hline \multirow[b]{2}{*}{ Type de la réaction } & \multicolumn{2}{|c|}{ Sensibilité } & \multirow[b]{2}{*}{ Fidélité } \\
\hline & $\begin{array}{c}\text { Par rapport } \\
\text { au lait cru } \\
\%\end{array}$ & $\begin{array}{c}\text { Par rapport } \\
\text { à } \mathrm{H}_{2} \mathrm{O}_{2} \\
\% \text { o }\end{array}$ & \\
\hline Dupoùy......... & 2 & 1 & Parfaite \\
\hline Storch $\ldots \ldots \ldots$ & 1 & 1 & Aberrances avec le lait \\
\hline Rotenfugser ...... & 1 & 0,5 & pasteurisé viejlli [1] \\
\hline Schern-Schellhaze . & 10 & 0,5 & $\begin{array}{l}\text { Aberrances dues à l'alté- } \\
\text { ration du réactif [2] }\end{array}$ \\
\hline
\end{tabular}

Ces résultats appellent les remarques suivantès:

Remarque I. Les aberrances avec le lait pasteurisé vieilli ont été signalées pour la réaction de Storch par Tноме́, le laboratoire Jorgensen [6], ORLA-Jensen et Winther [7] et confirmées par MASECK [8]. Le présent travail nous a permis de montrer que la réaction de Rotenfusser fournissait des indications sujettes aux mêmes erreurs d'interprétation.

Remarque II. Notre étude nous a montré que la réaction de Schern-Schellhaze pouvait donner lieu à l'apparition de résultats aberrants imputables à l'altération du réactif qui doit être utilisé dans les vingt-quatre heures suivant sa préparation. Tout se passe comme si l'altération du réactif se traduisait par la formation de traces de peroxyde. Il y a donc intérêt à faire dans tous les cas des témoins pour discriminer la coloration due à l'altération acciden- 
telle du réactif de la coloration imputable à la présence de peroxyde dans le lait.

Pour caractériser de faibles quantités de peroxyde, il nous faut choisir une réaction particulièrement sensible par rapport à l'eau oxygénée; ceci nous a conduits à éliminer les réactions de Storch et de Dupouy. D'autre part, le réactif ne devant pas être sensible aux modifications subies par le lait pasteurisé vieilli, nous avons dû éliminer la réaction de Rotenfusser.

Nous avons retenu, pour notre étude, la réaction de SchernSchellhaze. Cette réaction étant d'une pratique peu courante, nous rappelons ci-après la technique de son application :

Composition du réactif :

Gaïacol pur eristallisé $\ldots \ldots \ldots \ldots \ldots \ldots \ldots \ldots \ldots \ldots \ldots, 10$ grammes

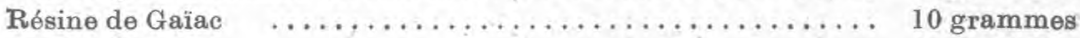
Alcool absolu .......................... $80 \mathrm{~cm}^{2}$

On filtre. Pour éviter l'altération rapide du réactif (remarque II), on prépare, d'une part, une solution de 10 grammes de gaïacol dans $40 \mathrm{~cm}^{3}$ d'alcool absolu et, d'autre part, une solution de 10 grammes de résine de gaïac dans $40 \mathrm{~cm}^{3}$ d'alcool absolu. Il suffit alors de mélanger à parties égales les deux solutions au moment de l'emploi.

La réaction positive se traduit par l'apparition d'une coloration bleu intense.

\section{Etude du comportement de l'eau oxygénée en mélange avec le lait}

Cette étude tendait à préciser la vitesse de décomposition de l'eau oxygénée dans le lait cru et dans le lait pasteurisé en s'adressant à une méthode sensible et fidèle. Nous avons retenu, à cet effet, la réaction de Schern-Schellhaze dont nous venons d'exposer la technique, en tenant compte des remarques ci-dessus mentionnées.

\section{$1^{\circ}$ Comportement de l'eau oxygénée dans le lait cru.}

Nous avons`ajouté à du lait cru, de l'eau oxygénée à 10 volumes à des doses comprises entre 2 et $4 \%$ et pratiqué d'heure en heure sa recherche.

Les résultats moyens de deux séries d'essais sont rassemblés dans le tableau II.

La méthode que nous employons permet de déceler l'eau oxygénée ajoutée à la dose de $2 \%$ après cinq heures de contact, et après sept heures pour la dose de $4 \%$. 
TABLEAU II

TEMPS MOYENS DE DÉCOMPOSITION DE L'EAU OXIGENEE DANS LE LAIT CRU EN FONCTION DES QUANTITÉS AJOUTEEES

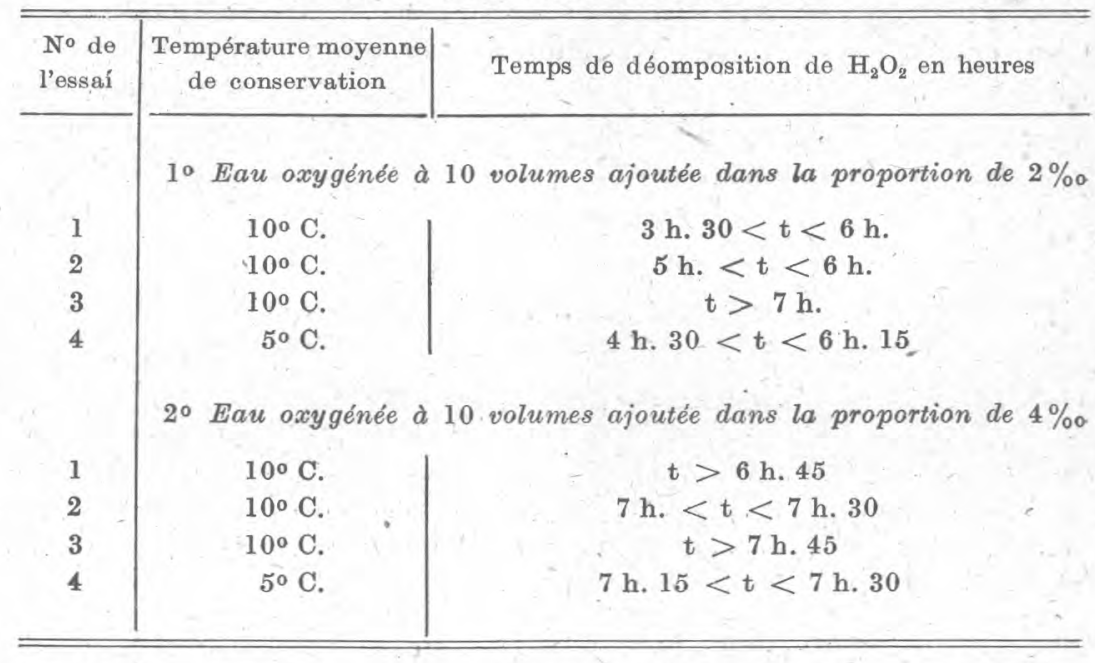

\section{$2^{\circ}$ Comportement de l'eau oxygénée dans le lait pasteurisé.}

Nous recherchons l'eau oxygénée dans le lait pasteurisé en le régénérant par addition de $10 \%$ de lait cru. Les résultats obtenus. sont groupés dans le tableau III.

\section{TABLEAU III}

TEMPS DE DÉCOMPOSITION DE L'EAU OXYGÉNÉE DANS LE LAIT PASTEURISE EN FONCTION DES QUANTITÉS AJOUTÉES

\begin{tabular}{|c|c|c|}
\hline $\begin{array}{l}\text { No de } \\
\text { l'essai }\end{array}$ & $\begin{array}{c}\text { Température moyenne } \\
\text { de conservation }\end{array} \mid$ & Temps de décomposition de $\mathrm{H}_{2} \mathrm{O}_{2}$ en heures \\
\hline & 10 Eau oxygénée à & 0 volumes ajoutée dans la proportion de $2 \%$ o \\
\hline 1 & $1^{\circ} \mathrm{C}$. & $\mathrm{t}>41 \mathrm{~h}$ \\
\hline 3 & $20^{\circ} \mathrm{C}$. & $40 \mathrm{~h} .<\mathrm{t}<48 \mathrm{~h}$ \\
\hline 3 & $10^{\circ} \mathrm{C}$. & $\mathrm{t}>22 \mathrm{~h} .15$ \\
\hline 4 & $5^{\circ} \mathrm{C}$ & $t>30 \mathrm{~h}$ \\
\hline 5 & 5 à $10^{\circ} \mathrm{C}$ & $t>30 \mathrm{~h}$ \\
\hline $\begin{array}{l}1 \\
2 \\
3 \\
4 \\
5\end{array}$ & $\begin{array}{c}2^{\circ} \text { Eau oxygénée à } \\
1^{\circ} \mathrm{C} . \\
20^{\circ} \mathrm{C} . \\
10^{\circ} \mathrm{C} . \\
5^{\circ} \mathrm{C} . \\
5 \text { à } 10^{\circ} \mathrm{C} .\end{array}$ & $\begin{array}{l}\text { volumes ajoutée dans la proportion de } 4 \% \text { o } \\
\qquad \begin{aligned} \mathrm{t} & >41 \mathrm{~h} \\
40 \mathrm{~h} . & <\mathrm{t}<48 \mathrm{~h} \\
\mathrm{t} & >30 \mathrm{~h} \\
\mathrm{t} & >30 \mathrm{~h} . \\
\mathrm{t} & >30 \mathrm{~h} .\end{aligned}\end{array}$ \\
\hline
\end{tabular}


Cette méthode très sensible permet de déceler avec une grande sûreté l'eau oxygénée ajoutée au lait pasteurisé à des doses comprises. entre 2 et $4 \%$ pendant les trente premières heures de contact. Dans un seul cas, la disparition de l'eau oxygénée est totale après. un contact de $22 \mathrm{~h}, 15$.

Signalons qu'on peut accroître légèrement la sensibilité de la. méthode en augmentant la quantité de lait cru ajouté au lait pasteurisé. Enfin, si les circonstances actuelles le permettait, il serait préférable de substituer au lait cru une solution concentrée en peroxydase pour la régénération du pouvoir peroxydasique du. lait pasteurisé.

Nous allons maintenant essayer de tirer parti sur le plan pratique de ces résultats.

\section{Contrôle pratique de l'addition frauduleuse d'eau oxygénée dans le lait de consommation}

Nous avons cherché, en nous appuyant sur les résultats précédents, à réaliser pratiquement un contrôle permettant de caractériser avec certitude l'eau oxygénée ajoutée au lait destiné à la consommation d'un grand centre.

\section{$1^{\circ}$ Cas du lait cru.}

Le contrôle est impossible pour le lait de la traite du soir livré à la consommation le lendemain matin seulement. Il est possible, par contre, pour la traite du matin dans le cas où la livraison est assez rapide. Le fait que ce contrôle ne peut s'appliquer au lait de la traite du soir montre qu'il n'est possible d'incorporer la recherche de l'eau oxygénée dans le contrôle des laits crus de consommation qu'en tenant compte des résultats positifs.

\section{$2^{\circ}$ Cas du lait pasteurisé.}

Notre expérimentation a porté sur le lait en provenance d'un atelier de pasteurisation situé à 165 kilomètres de Paris. A la laiterie, lors de la mise en pots, nous avons ajouté de l'eau oxygénée dans un certain nombre de pots. Des échantillons ont été prélevés sur ces pots en gare d'arrivée et la recherche a été effectuée selon la technique que nous avons décrite, environ vingt-quatre heures après la mise en pots. Parallèlement, nous avons fait une analyse bactériologique des échantillons.

Les résultats de ces essais sont rassemblés dans le tableau IV ci-après : 


\section{TABLEAU IV}

DETECTION AU STADE DE LA CONSOMMATION DE L'EAU OXIGENEE AJOUTEEE IMMEDIATEMENT APRESS PASTEURISATION AU DEPOT DE CAMPAGNE

\begin{tabular}{|c|c|c|c|c|}
\hline \multirow{2}{*}{$\begin{array}{l}\text { No de } \\
\text { l'essai }\end{array}$} & Proportion & \multirow{2}{*}{$\begin{array}{l}\text { Recherche } \\
\text { de } \mathrm{H}_{2} \mathrm{O}_{2}\end{array}$} & \multicolumn{2}{|c|}{$\begin{array}{l}\text { Dénombrements microbiens, germes } \\
\text { par centimètre cube }\end{array}$} \\
\hline & ajoutée & & Germes banaux & $\begin{array}{l}\text { Bactéries } \\
\text { coliformes }\end{array}$ \\
\hline & \multicolumn{4}{|c|}{ Première série: Lait standard conservé pendant le transport à $5^{\circ} C$. } \\
\hline Témoin & 0 & négative & 274.000 & 0,9 \\
\hline 1 & $1 \%$ & négative & 195.000 & 0 \\
\hline 2 & $-4 \%$ & positive & 160.000 & 0 \\
\hline & \multicolumn{4}{|c|}{ Deuxième série : Lait écrémé conservé pendant le transport à $5^{\circ} C$. } \\
\hline Témoin. & 0 & négative & & 6 \\
\hline 1 & $2 \% 0$ & positive & 158.000 & 0 \\
\hline 2 & $3 \%$ o & positive & 146.000 & 0 \\
\hline 3 & $4 \% 0$ & positive & 100.000 & 0 \\
\hline
\end{tabular}

L'analyse bactériologique montre l'effet inhibiteur de l'antiseptique employé à d'aussi faibles doses.

Ces résultats confirment ceux que nous avons obtenus au laboratoire lors de l'expérimentation et montrent que la recherche de l'eau oxygénée ajoutée frauduleusement au lait pasteurisé peut être pratiquée à l'arrivée au centre de consommation.

Nos essais ayant porté sur un dépôt expédiant son lait dans les conditions moyennes de l'ensemble du bassin laitier parisien, ce résultat peut être étendu sans risques d'erreurs à la totalité des dépôts envoyant du lait pasteurisé sur la capitale.

\section{Conclusions}

\section{Dans le cas des laits crus.}

Seuls les résultats positifs peuvent être valablement retenus, les résultats négatifs ne permettant pas de conclure avec certitude dès que la recherche est effectuée plus de cinq heures après la traite.

\section{$2^{\circ}$ Dans le cas des laits pasteurisés.}

Les indications du dénombrement microbien ne peuvent être considérées comme des normes de la valeur hygiénique des laits de consommation qu'à condition que soient vérifiés parallèlement :

a) L'absence de bactéries pathogènes par la vérification 
d'un chauffage effectif suffisant, dont l'action bactéricide n'a pas été compromise par une recontamination par du lait cru ou du lait mal pasteurisé. Cette vérification s'effectuera très simplement en mesurant le pouvoir peroxydasique du lait à analyser en s'adressant à une réaction fidèle et particulièrement sensible par rapport à la peroxydase, c'est-à-dire aux réactions de Storch ou de Dupouy ;

b) L'absence d'eau oxygénée dont l'action fausserait les résultats du dénombrement microbien tout en diminuant la valeur hygiénique intrinsèque du produit. Cette vérification sera aisément effectuée au moment' de l'analyse dans les délais normaux de livraison en utilisant la technique que nous indiquons dans cette étude.

\section{BIBLIOGRAPHIE}

[1] Tapernoux. Recherche de l'eau oxygénée dans le lait pasteurisé. Le Lait, 1928 , p. 410.

[2] Réaction de Dupouy. Sur les réactifs permettant de différencier le lait cru du lait pasteurisé ou bouilli. Gazette hebdomadaire des Sciences Médicales de Bordeaux, 1902, p. 566 .

[3] Réaction de Storch. 40e Rapport du Laboratoire d'Essais agricole de Danemark.

[4] Ancienne méthode de Rotenfusser (Article de Miethé et Witr. Détection par la peroxydase du lait ayant subi la pasteurisation haute. Molkerei Żeitung, t. XLVIII, 1934, p. 544-548.

[5] Schern et ScheldHazf. Berliner Tierärtaliche Wocherischrift, 1911, p. 868 .

[6] THоме́ et-Laboratoire Jorgensen (Article de ORLA-JENSEN et WINTHERR). La réaction de Storch. Le Lait, 1935, p. 247.

[7] OrLa-J ensen et Winther. La réaction de Storch. Le Lait, 1935, p. 247.

[8] Maseć. L'influence de l'âge du lait sur la sensibilité de la réaction de Storch. Le Lait, p. 941.

TENEUR DU LAIT DE LA VACHE EN VITAMINE P.P. * par

\section{MUle Madeleine MOREL et J. BARATTE}

La teneur en nicotinamide du lait de vache oscille, d'après les différents auteurs, entre $0 \mathrm{mgr}$. 1 et $0 \mathrm{mgr}$. 5 pour 100 grammes. On pouvait se demander si, chez la vache comme chez la femme (1), la richesse en vitamine $\mathrm{PP}$ du lait dépendait de celle du régime alimentaire.

Tous nos dosages ont été effectués d'après la technique sui-

(*) Annales de l'Institut Pasteur, 68, 1942, 538.

(1) Bibliographie in L. Justrn-Besançon et A. Lwofr. Vitamine antipellagreuse et Avitaminoses nicotiniques. Masson, édit., Pàris, 1942. 